\title{
CONVENCIONALIZACIÓN Y ADAPTACIÓN EN ESPAÑOL BONAERENSE. APORTE AL ESTUDIO DE LAS IMPORTACIONES LÉXICAS
}


El estudio de la neologización operada por la vía de la importación léxica conduce inevitablemente a atender al grado de convencionalización del uso de las piezas léxicas importadas, tanto como al de su adaptación. En este artículo nos ocupamos en particular de la alternancia entre configuraciones diferentes que suele apreciarse para una misma importación léxica. Sobre la base de datos correspondientes a la variedad bonaerense del español, proponemos una distinción entre tipos diversos de alternancia que puede ser de utilidad en las indagaciones referidas a la convencionalización.

PALABRAS ClaVe: importaciones léxicas, convencionalización, adaptación, alternancia, español bonaerense

The study of neologization by means of lexical importation leads inevitably to pay attention to the degree of conventionalization of the use of imported lexical units, as well as to their adaptation degree. In this paper, we focus on the alternation between different configurations that is usually observed for the same lexical importation. On the basis of data from Buenos Aires Spanish, we propose a distinction between different types of alternation which can be useful for research concerning conventionalization.

KEY wORDS: lexical importations, conventionalization, adaptation, alternation, Buenos Aires Spanish 


\section{CONVENCIONALIZACIÓN Y ADAPTACIÓN EN ESPAÑOL BONAERENSE. APORTE AL ESTUDIO \\ DE LAS \\ IMPORTACIONES LÉXICAS}

Yolanda Hipperdinger UNSur-CONICET (Argentina)

\section{Introducción}

Entre los emergentes del contacto interlingüístico, el que más atención ha recibido por su general constatación, incluso en casos de contacto distante (véase Winford, 2003: 26), es el del trasvase de elementos léxicos de una lengua a otra. Esas importaciones de "forma y significado" (Gómez Molina, 2000: 328) ${ }^{1}$ constituyen una importante fuente de

\footnotetext{
${ }^{1}$ En relación con la "omnipresencia" del trasvase léxico referido y el sostenido interés por ellas se halla la coexistencia, en el acercamiento especializado, de numerosos criterios de detección y clasificación, así como de designaciones
} 
renovación y acrecentamiento léxico para la lengua que las recibe. Así, al referirse al neologismo Alvar Ezquerra (2005: 3) subraya la necesidad de "que el léxico se renueve, [...] por lo que son necesarias las palabras nuevas", y cuenta entre esas "palabras nuevas" las importaciones provenientes de otras lenguas que, al igual que las innovaciones obtenidas por la aplicación de recursos nativos, pierden su "novedad" a medida que se difunden.

En el caso de las importaciones léxicas, empero, la pérdida de la "novedad" no se vincula solo con la difusión, como lo señala el mismo autor en relación con diversas importaciones operadas en el español: jaula o jamón son tan exógenas como software, "pero el tiempo que llevan en la lengua no es el mismo, por lo que su adaptación al sistema (fónico y gráfico) no es el mismo, como tampoco es igual el sentimiento de los hablantes: las primeras no son sentidas como voces extranjeras, mientras que la última sí" (Alvar Ezquerra, 2005: 4). Establece de ese modo, por un lado, un vínculo causal entre la antigüedad (y consiguiente difusión) de una pieza léxica importada y su ajuste a las pautas de la lengua que la recibe; por otro lado (y lo que nos interesa destacar

diferentes. Aunque su discusión queda fuera de los propósitos de este artículo - pueden consultarse al respecto la esclarecedora revisión ofrecida por Gómez Capuz (1998) y, entre otras presentaciones recientes, la de De Baere (2012: 15 ss.) - , dejamos constancia de que decidimos emplear la denominación hiperonímica importaciones léxicas, filiada en la obra pionera de Haugen (1950: 214), por cuanto simultáneamente permite (a) distinguir de modo transparente el fenómeno del ingreso en "forma y significado" de otros fenómenos vinculados, en especial el del calco, y (b) reservar el (controversial pero) generalizado rótulo de préstamo para ingresos léxicos extendidos en el uso en la lengua receptora, i.e. de acuerdo con su acepción más corriente (véase Haspelmath, 2009: 41-42). Por el mismo uso técnico, véase e.g. T’Sou (2001). 
aquí), plantea la exigencia de una completa adaptación, en los usos orales $y$ en los escritos, para que los hablantes ya no reconozcan la extranjeridad de una importación léxica.

En relación con esto último ya Seco (1977: 197), por ejemplo, distinguía "las voces extranjeras que el idioma ha asimilado totalmente a su sistema" de "las palabras que en su grafía, o en su pronunciación, o en ambas cosas a la vez, acusan en los hablantes una conciencia de que emplean una palabra extranjera", destacando que el mantenimiento mismo de rasgos exógenos, en los usos orales $o$ en los escritos, constituye un indicador de que los hablantes reconocen aún la extranjeridad de la pieza importada.

De acuerdo con extendidas posiciones como las presentadas, entonces, un elemento léxico importado "se despoja de su carácter neológico" (Alarcos Llorach, 1992: 21) cuando su extranjeridad deja de ser reconocible, ${ }^{2}$ lo cual abarca dos cuestiones ligadas, aunque diferentes, en la medida en que implica

a) tanto que no haya fluctuación en ningún canal entre configuraciones alternativas ${ }^{3}$

\footnotetext{
${ }^{2}$ Una distinción recurrente en la bibliografía especializada, sostenida también sobre la diferencia entre las importaciones léxicas que resultan reconocibles (o no) como tales para los usuarios, es la que opone las patentes a las no patentes (véanse e.g. Miotti, 2008: 273 y Sanou et al., 2012). No obstante, también puede encontrarse la denominación de no patente aplicada a influjos verificables en fenómenos diferentes de la importación de "forma y significado", como la extensión semántica (véase e.g. Gómez Capuz, 2004: 13).

${ }^{3}$ La fluctuación referida ha sido señalada en diversas situaciones de contacto. Entre las que involucran al español, para el caso de la oralidad Torres Cacoullos y Vigil (2006: 4), por ejemplo, la observan como "realización variable de la misma voz" en palabras provenientes del inglés en el sudoeste de los Estados Unidos; para el caso de la escritura, sirve como ejemplo la afirmación de Corbella (1997: 41) de que los "galicismos patentes" en español son fácilmente recono-
} 
b) como que, además, las configuraciones seleccionadas no sean reconocibles como exógenas por su forma.

La necesidad de abordar por separado las cuestiones relativas a la fijación o convencionalización ${ }^{4}$ y a la adaptación se aprecia fácilmente si se enfocan importaciones léxicas que no han perdido su "carácter neológico" en el sentido señalado.

Por una parte, puede operarse la selección social de una configuración no (o no completamente) adaptada para una importación léxica. La actualización de esa posibilidad se registra sobre todo en la escritura, libre de la presión que los patrones articulatorios ejercen en la oralidad. ${ }^{5}$ En español, un claro ejemplo lo provee la decidida preferencia por el mantenimiento de la representación de origen, piz$z a$, para esa difundida importación léxica del italiano (véase e.g. Janíčková, 2008: 30, 52). Casos como este demuestran que puede convencionalizarse el uso de una configuración no adaptada, i.e. que puede haber convencionalización sin adaptación.

cibles por la fluctuación de su configuración grafémica (e.g. "boutique / butique / butic").

${ }^{4}$ Entendemos por convencionalización, en consonancia con Backus (2003: 244), el consenso social sobre el uso de una forma, lo que en casos de diferencia interlingüística releva al hablante de tener que decidir qué lengua tomará como modelo. Aunque para las importaciones léxicas tal convencionalización puede eventualmente coincidir con una fijación normativa, es de destacar que pueden operarse consensos sobre el uso en ausencia de esta última, así como que, existiendo formas normativizadas, las formas privilegiadas por el uso pueden no coincidir con ellas.

${ }^{5}$ Es por ello que, como lo señala Castillo Carballo (2011: 64), “resulta más fácil el consenso fonético que el ortográfico". Por la innecesariedad de la "adaptación ortográfica” a los fines comunicativos, véase Haspelmath (2009: 42). 
Por otra parte, y en sentido inverso, puede haber también adaptación sin convencionalización. La actualización de esa posibilidad se aprecia en especial en la oralidad, en el marco del contacto indirecto, cuando el productor individual monolingüe se enfrenta a la necesidad de realizar oralmente importaciones léxicas que encuentra escritas (por ejemplo en inscripciones en productos comerciales o publicidades) pero que no se hallan difundidas en el uso, por lo que no ha podido operarse la selección social de una configuración determinada que le sirva como guía. Ese hablante puede "leer en español" la importación de que se trate, o intentar alguna imitación de la pronunciación de origen recurriendo a correspondencias grafémico-fonológicas diferentes de las de su lengua, y miméticas respecto de las de la lengua de origen, que pueda haber inferido de la realización de importaciones léxicas en uso procedentes de la misma lengua donante. ${ }^{6}$ La primera "solución" es adaptativa, como es obvio, en la medida en que no interviene más conocimiento que el de la relación entre grafía y oralidad establecida en la lengua receptora, pero también la segunda supone adaptación, ya que genera realizaciones que solo parcialmente se acercan a las de origen.

Lo expuesto ratifica que la convencionalización y la adaptación constituyen dimensiones analíticas diferentes, y muestra simultáneamente que corresponde también

\footnotetext{
${ }^{6}$ Esas opciones se corresponden con las identificadas en el clásico trabajo de Pratt (1980: 136 et alibi) sobre el anglicismo español: la "pronunciación española de la grafía inglesa" y la "imitación española de la pronunciación inglesa”. Por la autonomización de correspondencias grafémico-fonológicas miméticas, véanse Gómez Capuz (2001) y Hipperdinger (2013a).
} 
abordarlas por separado en los usos orales y en los escritos de las piezas importadas. Además, y especialmente, lo que llevamos dicho conduce a concluir que la atención a la alternancia debe ser central en el estudio de las importaciones léxicas: para determinar si se ha operado convencionalización en el uso de una pieza léxica importada, en uno u otro canal, es necesario como mínimo verificar si existe o no alternancia entre configuraciones diferentes, y este es a su vez el paso previo al análisis del grado de adaptación de la(s) configuración(es) registrada(s).

En este trabajo nos ocuparemos en particular de dicha alternancia, con el objetivo de ensayar un aporte procedimental a su abordaje. Nos basaremos para ello en datos empíricos correspondientes a la variedad bonaerense del español de la Argentina, hablada en la capital y su zona de influencia. ${ }^{7}$ A partir del análisis de los resultados de un reciente relevamiento de las configuraciones escritas de importaciones léxicas en el área léxica de la gastronomía, ${ }^{8}$ procuraremos mostrar que es posible distinguir tipos diversos de alternancia, atendiendo a la adaptación de las configuraciones coexistentes, y que la distinción que proponemos posee valor heurístico respecto de la convencionalización. ${ }^{9}$

\footnotetext{
${ }^{7}$ En relación con la denominación escogida, así como con la caracterización del español bonaerense, véase Fontanella de Weinberg $(1987,2000)$.

${ }^{8}$ Por la especial permeabilidad de esa área léxica a la neologización por la vía de la importación, véase e.g. Romaine (1995: 142).

${ }^{9}$ Una primera formulación de la propuesta contenida en este artículo fue expuesta en Hipperdinger (2013b).
} 


\section{Importaciones léxicas en español bonaerense: alternancia en la escritura}

Presentamos seguidamente los resultados obtenidos, en relación con la alternancia en la que centramos nuestra atención, en el relevamiento que recientemente realizamos en setenta locales comerciales de expendio de comidas elaboradas, ubicados en el centro y macrocentro de la ciudad de Bahía Blanca. ${ }^{10}$ En la publicitación realizada en ellos de las especialidades ofrecidas - que suele abarcar el detalle de sus ingredientes principales - registramos el uso de 123 importaciones léxicas, para 63 de las cuales constatamos configuraciones alternativas. De entre estas últimas, seleccionamos para la confección del siguiente cuadro las piezas léxicas registradas en un mínimo de diez de los locales comerciales referidos. El cuadro remite a 1089 registros, para 29 importaciones léxicas de diversa difusión en el uso. Las ingresamos por los respectivos alternantes de empleo mayoritario, siguiendo un orden alfabético, y para cada importación precisamos el número de registros de cada alternante, ordenándolos por su frecuencia de aparición.

\footnotetext{
${ }^{10}$ Bahía Blanca (nuestro lugar de residencia) cuenta con alrededor de 300,000 habitantes y se ubica a 700 kilómetros al sur de la Ciudad Autónoma de Buenos Aires, capital de la Argentina. Pertenece a su misma región dialectal, "y no resulta posible a primera vista distinguir lingüísticamente a los hablantes de una y otra ciudad" (Fontanella de Weinberg et al., 1991: 36).
} 


\begin{tabular}{|c|c|c|}
\hline & $\begin{array}{l}\text { Número total de registros } \\
\text { en el corpus }\end{array}$ & $\begin{array}{l}\text { Configuraciones } \\
\text { registradas }\end{array}$ \\
\hline baguette & 26 & $\begin{array}{l}\text { baguette (19) } \\
\text { baguett ( } 4) \\
\text { bagette (3) }\end{array}$ \\
\hline bolognesa & 29 & $\begin{array}{l}\text { bolognesa }(22) \\
\text { boloñesa }(7)\end{array}$ \\
\hline brochette & 21 & $\begin{array}{l}\text { brochette (15) } \\
\text { brochett (3) } \\
\text { brocheta (3) }\end{array}$ \\
\hline brownie & 19 & $\begin{array}{l}\text { brownie }(18) \\
\text { brawnie (1) }\end{array}$ \\
\hline bruschetta & 14 & $\begin{array}{l}\text { bruschetta }(12) \\
\text { bruscheta }(2)\end{array}$ \\
\hline calzone & 11 & $\begin{array}{l}\text { calzone }(10) \\
\text { calzzone }(1)\end{array}$ \\
\hline cappelletti & 21 & $\begin{array}{l}\text { cappelletti (6) } \\
\text { cappellettis (3) } \\
\text { cappeletis ( } 3 \text { ) } \\
\text { capelleti (3) } \\
\text { capelletti (2) } \\
\text { capellettis (2) } \\
\text { capelletis (2) }\end{array}$ \\
\hline cappuccino & 34 & $\begin{array}{l}\text { cappuccino }(13) \\
\text { capuchino }(8) \\
\text { capuccino }(7) \\
\text { cappucino }(3) \\
\text { capucino }(3)\end{array}$ \\
\hline capresse & 41 & $\begin{array}{l}\text { capresse }(24) \\
\text { caprese }(16) \\
\text { caprisse }(1)\end{array}$ \\
\hline champignon & 57 & $\begin{array}{l}\text { champignon (42) } \\
\text { champiñón (13) } \\
\text { champigñon }(2)\end{array}$ \\
\hline chip & 20 & $\begin{array}{l}\text { chip (17) } \\
\text { chipp (3) }\end{array}$ \\
\hline ciboulette & 17 & $\begin{array}{l}\text { ciboulette (14) } \\
\text { ciboullette (2) } \\
\text { ciboullete (1) }\end{array}$ \\
\hline
\end{tabular}


Yolanda Hipperdinger

\begin{tabular}{|c|c|c|}
\hline crêpe & 19 & $\begin{array}{l}\text { crêpe }(7) \\
\text { creppe }(6) \\
\text { crepe }(5) \\
\text { crepé }(1)\end{array}$ \\
\hline filetto & 17 & $\begin{array}{l}\text { filetto (16) } \\
\text { filletto (1) }\end{array}$ \\
\hline fugazza & 28 & $\begin{array}{l}\text { fugazza (26) } \\
\text { fugaza (2) }\end{array}$ \\
\hline fugazzeta & 23 & $\begin{array}{l}\text { fugazzeta (14) } \\
\text { fugazzetta ( } 8) \\
\text { fugazeta (1) }\end{array}$ \\
\hline fusilli & 22 & $\begin{array}{l}\text { fusilli (9) } \\
\text { fusiles (4) } \\
\text { fuccilli (3) } \\
\text { fuccili (2) } \\
\text { fuchilli (2) } \\
\text { fuchili (1) } \\
\text { fuscilli (1) }\end{array}$ \\
\hline grillé & 28 & $\begin{array}{l}\text { grillé (14) } \\
\text { grille (8) } \\
\text { grillado/a (6) }\end{array}$ \\
\hline lasagna & 30 & $\begin{array}{l}\text { lasagna (19) } \\
\text { lasaña (6) } \\
\text { lasagña (3) } \\
\text { lazagna (2) }\end{array}$ \\
\hline mozzarella & 278 & $\begin{array}{l}\text { mozzarella }(148) \\
\text { muzzarella }(121) \\
\text { mozarella }(4) \\
\text { muzzarela }(3) \\
\text { muzarella }(2)\end{array}$ \\
\hline noisette & 10 & $\begin{array}{l}\text { noisette }(7) \\
\text { noicette }(2) \\
\text { noissette }(1)\end{array}$ \\
\hline pizzeta & 16 & $\begin{array}{l}\text { pizzeta (15) } \\
\text { pizzetta (1) }\end{array}$ \\
\hline ricotta & 69 & $\begin{array}{l}\text { ricotta }(38) \\
\text { ricota }(31)\end{array}$ \\
\hline roquefort & 92 & $\begin{array}{l}\text { roquefort (91) } \\
\text { roqueford (1) }\end{array}$ \\
\hline
\end{tabular}




\begin{tabular}{|l|c|l|}
\hline sandwich & 90 & $\begin{array}{l}\text { sandwich (66) } \\
\text { sándwich (24) }\end{array}$ \\
\hline spaghetti & 23 & $\begin{array}{l}\text { spaghetti (15) } \\
\text { spaghettis (3) } \\
\text { spaguetti (3) } \\
\text { espaguetis (2) }\end{array}$ \\
\hline tagliatelle & 10 & $\begin{array}{l}\text { tagliatelle (7) } \\
\text { tagliattelle (3) }\end{array}$ \\
\hline tiramisú & 13 & $\begin{array}{l}\text { tiramisú (10) } \\
\text { tiramisu (2) } \\
\text { tiramizzu (1) }\end{array}$ \\
\hline tortelletti & 11 & $\begin{array}{l}\text { tortelletti (4) } \\
\text { tortelletis (3) } \\
\text { torteletis (3) } \\
\text { tortellettis (1) }\end{array}$ \\
\hline
\end{tabular}

Lo primero que se observa es la prevalencia de alternantes no adaptados entre los mayoritarios para las importaciones léxicas consignadas. $\mathrm{Al}$ respecto, cabe señalar que, en cambio, cuando registramos una única configuración escrita para una pieza léxica importada se trató tanto de formas no adaptadas como de formas adaptadas, incluso en el caso de importaciones igualmente difundidas y procedentes de la misma lengua como pizza y panceta, de 88 y 58 apariciones en el corpus, respectivamente.

Por otra parte, salta a la vista que es variable el número de alternantes registrado para las distintas piezas léxicas: en el referido relevamiento llega a siete en importaciones del italiano empleadas en plural (cappelletti $\sim$ cappellettis cappeletis $\sim$ capellet $i \sim$ capelletti $\sim$ capellettis $\sim$ capelletis y fusilli $\sim$ fusiles $\sim$ fuccilli $\sim$ fuccili $\sim$ fuchilli $\sim$ fuchili $\sim$ fuscilli), y a cinco entre las empleadas en singular (cappuccino capuchino $\sim$ capuccino $\sim$ cappucino $\sim$ capucino y mozzarella $\sim$ muzzarella $\sim$ mozarella $\sim$ muzzarela $\sim$ muzarella). Los al- 
ternantes, además, presentan frecuencias relativas dispares: frente al carácter idiosincrásico de brawnie, calzzone o roqueford, boloñesa no es raro, y ricota directamente compite de cerca con ricotta.

Por último, puede observarse que los alternantes registrados para una misma importación léxica implican en algunos casos opciones diferentes en relación con la adaptación (así, lasagna se ajusta a la forma de origen y lasaña al español) y en otros casos no (por ejemplo, los alternantes baguett y bagette no reproducen estrictamente la configuración grafémica de origen pero han sido generados por el intento de hacerlo, como lo muestra la conservación de $t t$ ).

En principio puede pensarse que, cuanto mayor es el número de configuraciones relevadas para una misma pieza léxica, menor es el consenso social existente respecto de su uso en relación con la adaptación. Sin embargo, los datos no avalan estrictamente esa suposición: si bien la alternancia que nos interesa se vincula siempre con inseguridad lingüística, la fuente de esa inseguridad puede diferir.

Así, la primera observación que realizamos se vincula con el hecho de que, si los hablantes no se sienten inseguros en su producción cuando el consenso social ha enfocado para una cierta importación léxica una forma adaptada, el desconocimiento de la lengua donante posibilita dudas cuando el target es la configuración de origen. Es el caso, por ejemplo, de la alternancia mozzarella $\sim$ muzzarella $\sim$ mozarella $\sim$ muzzarela $\sim$ muzarella, en la que todos los alternantes conservan al menos una de las digrafías de origen, o de capresse $\sim$ caprese $\sim$ caprisse, en la que el intento de apegarse a la configuración de origen se evidencia por la exageración 
de la extranjeridad que supone la duplicación de $s$. Si todos los alternantes registrados tienden a un mismo modelo, independientemente de su número, claramente sirven como indicadores de que la preferencia social por una cierta configuración formal está definida en cuanto a la adaptación: esos alternantes "aproximados" son el resultado de una decisión convergente. ${ }^{11}$

La alternancia registrada entre configuraciones que representan opciones diferentes respecto de la adaptación, por el contrario, puede indicar la inexistencia de una preferencia social definida. En relación con ello, no obstante, cabe también la posibilidad de que solo una configuración se halle en uso por el conjunto de los hablantes para una pieza léxica importada pero que, distanciándose de la opción esperable o no marcada, se hagan ocasionalmente elecciones marcadas. ${ }^{12}$ Por ejemplo, quien conozca la forma de origen de una importación léxica cuyo uso se ha convencionalizado bajo una configuración adaptada podrá "desadaptarla" si desea hacer gala de virtuosismo lingüístico o mostrarse como conocedor. Aunque el efecto rupturista de tal elección (relevante desde una perspectiva interaccional) es en el marco de la argumentación que desarrollamos solo un indicador de consenso, sirve igualmente para mostrar que el registro de configuraciones que impliquen opciones diferentes respecto de la adaptación es solo una condición necesaria para concluir

\footnotetext{
${ }^{11}$ Particularmente sintomáticos en tal sentido son los alternantes hipercorrectos, como los referidos capresse $\sim$ caprisse, que constatamos reiteradamente en nuestro relevamiento (calzzone, chipp, ciboullette, filletto, etc.). Para un estudio específico sobre formas grafémicas hipercorrectas, realizado sobre las mismas región dialectal y área léxica que el presente artículo, véase Hipperdinger (2013c).

${ }^{12}$ La noción de marcación que empleamos extrapola la contribución de MyersScotton (1993) sobre el cambio de código a la alternancia que aquí nos interesa.
} 
que no se ha operado convencionalización. La condición suficiente la constituye la frecuencia relativa de tales alternantes: de ella depende tanto el aislamiento de los empleos idiosincrásicos como la determinación de en qué medida compiten en el uso configuraciones que procuran ajustarse al modelo de la lengua donante o a las pautas de la lengua receptora.

\section{Alternancia centrípeta y centrífuga}

Como ha podido apreciarse, tanto la convencionalización como la adaptación implican cuestiones de grado. Entendemos, en consecuencia, que pueden conceptualizarse (para cada canal) como sendas escalas continuas:

a) sobre la escala de la adaptación se ubicarán las distintas configuraciones con las que ocurre una cierta importación léxica, mientras que

b) sobre la escala de la convencionalización se ubicarán las importaciones léxicas mismas, de acuerdo con el grado de consenso social que se verifique respecto del uso de cada una con una cierta configuración.

En la escala de la adaptación, constituirá el polo negativo la reproducción de la configuración de origen, y el polo positivo la configuración más adaptada que sea posible a las pautas de la lengua receptora. ${ }^{13}$ En la escala de la convencionalización constituirá el polo positivo el uso exclusivo de una única configuración para una cierta pieza léxica, y

\footnotetext{
13 Obviamente, para cada importación léxica la cantidad de posibilidades de adaptación incompleta dependerá, a su vez, del grado de distancia formal entre las configuraciones que constituyan los polos de la escala.
} 
el polo negativo el empleo de configuraciones alternativas que no solo impliquen opciones diferentes en relación con la adaptación sino que, además, presenten una frecuencia de uso equiparable. ${ }^{14}$

Si se grafica la escala de la adaptación, los alternantes que derivan de una decisión convergente se ubicarán de modo arracimado; los alternantes que se releven como indicadores de opciones diferentes, en cambio, se dispersarán. Por ello, proponemos llamar centrípeta a la alternancia que deriva de una decisión convergente, en la medida en que los alternantes tienden al "centro" constituido por el modelo, y centrifuga a la que implica opciones diferentes. ${ }^{15}$

Para dos de las importaciones léxicas enlistadas, que transparentan esta diferencia, tal graficación puede presentarse como sigue:

Alternancia centrípeta:

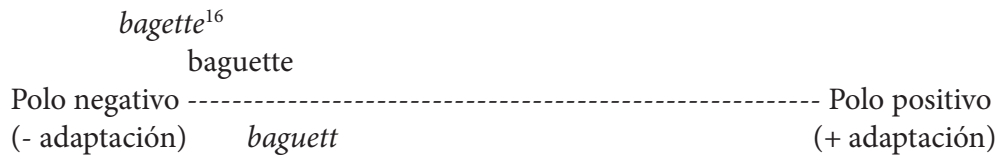

\footnotetext{
${ }^{14}$ Para una conceptualización similar, pero aplicada a una situación que -en los términos de Thomason y Kaufman (1988) - no se abarca bajo el signo del préstamo a una lengua desde otra sino bajo el de la interferencia de una a otra, véase Elizaincín (1992: 67ss.).

${ }^{15}$ En otros campos disciplinares se ha recurrido a los mismos usos metafóricos. Véanse e.g. Wallon (1941) y Sartori (1982).

${ }^{16}$ En la graficación buscamos reflejar que la forma hipercorrecta bagette - en la que fue suprimida la $u$ que en francés se requiere, en coincidencia con la ortografía española, para la representación de /g/ ante vocal anterior- muestra la opción por no adaptar de un modo más decidido aún que la forma que reproduce la de origen, cuya selección puede deberse exclusivamente a la "costumbre" que la convencionalización supone.
} 
Alternancia centrífuga:

champignon

champigñon

champiñón

(- adaptación)

- Polo positivo

(+ adaptación)

Los dos tipos de alternancia que distinguimos pueden vincularse, de acuerdo con nuestros datos, con diferentes fuentes de inseguridad lingüística:

a) la alternancia centrípeta se relaciona con la inseguridad de quien habla o escribe respecto de "cómo es" - modo este el estereotipado en la comunidad bajo estudio para referir al modelo exógeno- la correspondiente configuración de origen;

b) la alternancia centrífuga, en cambio, se relaciona con la inseguridad de quien habla o escribe respecto de qué configuración -en principio, adaptada o no-conviene elegir en ausencia de una preferencia social definida.

La distinción entre ambos tipos de alternancia tiene valor heurístico, por cuanto se podrá afirmar que existe consenso social aun cuando se verifique alternancia si esta es centrípeta.

Es también posible, según lo muestran los resultados de nuestro relevamiento, que ambos tipos de alternancia se verifiquen conjuntamente. Constituyen un ejemplo de ello las representaciones grafémicas alternativas cappuccino capuccino $\sim$ cappucino $\sim$ capucino $\sim$ capuchino. Cappuccino se ubica en el polo negativo de la escala de la adaptación, y capuchino en el polo positivo. La "españolización" se manifiesta en esta última forma tanto en la simplificación de la digrafía de origen para la consonante geminada como en la 
selección de ch para la representación grafémica de la consonante africada con la que se registra regularmente en la oralidad. En la medida en que la geminación aludida no tiene correlato en la lengua receptora, su simplificación resulta menos sintomática de la voluntad de adaptación que la representación grafémica de la africada, en la que claramente se opta por el modelo italiano $o$ por el español. Tomados los alternantes en conjunto, van de polo a polo en la escala de la adaptación, i.e. se dispersan sobre ella; la alternancia es por lo tanto centrífuga. No obstante, los alternantes que no "españolizan" se arraciman sobre el polo negativo de la escala de la adaptación, por lo que esa alternancia cabe en la definición de la que llamamos centrípeta.

En la escala de la adaptación, la ubicación de los alternantes constatados para la referida importación léxica del italiano podría presentarse del siguiente modo:

$\begin{array}{lr}\text { cappuccino capucino } & \text { capuchino } \\ \begin{array}{l}\text { Polo negativo - } \\ \text { (- adaptación) }\end{array} & \begin{array}{l}\text { cappucino } \\ \text { capuccino }\end{array}\end{array}$

Encontrar simultáneamente alternancia de ambos tipos para una importación léxica, como en el ejemplo comentado, puede ser común (en nuestro relevamiento, es también por ejemplo el caso de brochette y brochett frente a la adaptación representada por brocheta, entre las importaciones empleadas en singular, o de spaghetti(s) y spaguetti frente la máxima adaptación registrada para la pieza, espaguetis, entre las empleadas en plural). 
Las conclusiones de mayor relevancia respecto de la convencionalización, sin embargo, se derivan de la constatación de alternancia centrífuga, y es por lo mismo la que entendemos que corresponde enfocar procedimentalmente: si se la verifica para una importación léxica podrá hipotetizarse que no se ha operado convencionalización sobre su uso. El grado de competencia efectiva entre los alternantes que indican opciones diferentes, medido en términos de su frecuencia relativa, permitirá ulteriormente la ubicación de la pieza léxica sobre la escala pertinente.

Si se grafica la escala de la convencionalización, así, sobre la base de los datos consignados podrían ubicarse como sigue, por ejemplo, algunas de las importaciones léxicas que analizamos (referidas por su configuración mayoritaria):

\begin{tabular}{lcc}
\multicolumn{1}{c}{ ricotta cappuccino } & baguette \\
Polo negativo - & convitivo \\
(- convencionalización) & (+ convencionalización)
\end{tabular}

En el caso de la alternancia ricotta ricota, el alternante que reproduce la forma de origen sin adaptación es solo ligeramente mayoritario ( $55.07 \%$ de los registros); la fluctuación registrada para la pieza léxica es, por lo mismo, especialmente alta, por lo que decididamente tiende al polo negativo de la escala de la convencionalización. En el caso de cappuccino y las restantes configuraciones grafémicas registradas, la competencia se plantea entre la forma adaptada y la alternancia centrípeta en torno del modelo de la lengua donante; el alternante adaptado es minoritario $23.53 \%$ de los registros), por lo que la pieza se orienta al polo positivo de la escala, aunque dista de ubicarse sobre él. En ese polo, 
en cambio, se ubica la importación mayoritariamente escrita baguette, ya que los demás alternantes registrados constituyen igualmente intentos de reproducir la configuración de origen.

\section{Síntesis y conclusiones}

Nos ocupamos en este artículo de las cuestiones de convencionalización y adaptación implicadas en el estudio de las importaciones léxicas, enfocando en particular la alternancia por cuanto en ella se imbrican aspectos relativos a ambas.

En primer término, procuramos demostrar que la mera constatación de alternancia no alcanza para inferir que no se ha operado consenso social sobre el uso de una pieza léxica importada: para que pueda indicar falta de convencionalización de uso, los alternantes deben obedecer a opciones diferentes respecto de la adaptación.

Sobre esa base, distinguimos esta última alternancia, que denominamos centrífuga, de la constatada cuando los alternantes registrados constituyen todos actualizaciones de la misma opción, que llamamos centrípeta.

Si se registra exclusivamente alternancia centrípeta, puede inferirse que la opción en relación con la adaptación está socialmente definida. Si ambos tipos de alternancia se verifican conjuntamente para una importación léxica, la centrífuga es la que brinda la información de mayor relevancia respecto de la convencionalización: la alternancia centrípeta asume su rol indicial, de signo contrario, solo en ausencia de alternancia centrífuga. Las precisiones sobre el grado de 
convencionalización del uso de una importación léxica para la que se constate alternancia centrífuga, finalmente, dependerán de la frecuencia relativa con la que se registren las diferentes opciones en relación con la adaptación.

Obviamente, el acercamiento que proponemos admite mayor refinamiento, y la ratificación de su valor heurístico exige su aplicación a otros cuerpos de datos. Prevemos, en tal sentido, desarrollos futuros de nuestra investigación.

\section{Bibliografía}

Alarcos Llorach, Emilio (1992), “Consideraciones sobre el neologismo", en Javier María Pascual (ed.), El neologismo necesario, Madrid, Fundación EFE, pp. 19-29.

Alvar Ezquerra, Manuel (2005), El neologismo. Concepto, formación y aceptabilidad, Madrid, Liceus.

BACKus, AD (2003), "Can a mixed language be conventionalized alternational codeswitching?", en Yaron Matras y Peter Bakker (eds.), The mixed language debate. Theoretical and empirical advances, Berlín, Mouton / de Gruyter, pp. 237-270.

Castillo Carballo, María Auxiliadora (2011), "Voces extranjeras; de la realidad lingüística peninsular a la americana”, Itinerarios, 13, pp. 63-84.

Corbella, Dolores (1997), "Elementos para un análisis del préstamo francés en el español actual", en Manuel Almeida y Josefa Dorta (eds.), Contribuciones al estudio de la lingüística hispánica, tomo II, Madrid, Montesinos, pp. 39-47. 
De BAere, Jozefien (2012), Los extranjerismos en el español contemporáneo, Gent, Universiteit Gent.

Elizaincín, Adolfo (1992), Dialectos en contacto. Español y portugués en España y América, Montevideo, Arca.

Fontanella de Weinberg, María Beatriz (1987), El español bonaerense. Cuatro siglos de evolución lingüística (1580-1980), Buenos Aires, Hachette.

(2000), “El español bonaerense", en María Beatriz Fontanella de Weinberg (coord.), El español de la Argentina y sus variedades regionales, Buenos Aires, Edicial, pp. 37-61.

- Mercedes Blanco, Yolanda Hipperdinger, Elizabeth Rigatuso, Silvia Suardíaz de Antollini y Ana VIRKel (1991), Lengua e inmigración. Mantenimiento y cambio de lenguas inmigratorias, Bahía Blanca, Universidad Nacional del Sur.

Gómez Capuz, Juan (1998), El préstamo lingüístico. Conceptos, problemas y métodos, València, Universitat de València.

_ (2001), "Estrategias de integración fónica de los anglicismos en un corpus de español hablado: asimilación, compromiso y efectos estructurales", Estudios de Lingüística de la Universidad de Alicante, 15, pp. 1-85. - (2004), La inmigración léxica, Madrid, Arco Libros.

Gómez Molina, José Ramón (2000), “Transferencia y cambio de código en una comunidad bilingüe. Área metropolitana de Valencia (I y II)", Contextos, XVII-XVIII, pp. 309-360.

Haspelmath, Martin (2009), "Lexical borrowing: concepts and issues", en Martin Haspelmath y Uri Tadmor 
(eds.), Loanwords in the world's languages. A comparative handbook, Berlín, Walter de Gruyter, pp. 35-54.

Haugen, Einar (1950), "The analysis of linguistic borrowing", Language, 26, pp. 210-231.

HipPERDINGER, Yolanda (2013a), "Incorporaciones léxicas en el español de la Argentina. Sobre la adaptación de las adopciones", Forma y Función, 26 (2) (en prensa). (2013b), "Sobre el tratamiento de las importaciones léxicas. Alternancia centrípeta y centrifuga", $V$ Jornadas de Investigación en Humanidades, Bahía Blanca, Universidad Nacional del Sur.

_ (2013c), "Estereotipos que no simplifican. Hipercorrección grafémica en importaciones léxicas", en Yolanda Hipperdinger (comp.), Lenguas: conceptos y contactos, Bahía Blanca, EdiUNS (en prensa).

JaníčKovÁ, KRISTÝna (2008), Préstamos en la gastronomía, Brno, Masarykova Univerzita.

Miotti, Renzo (2008), "La pronunciación de los anglicismos técnicos en dos lenguas de especialidad", en Carmen Navarro, Rosa Rodríguez Abella, Francesca Dalle Pezze y Renzo Miotti (eds.), La comunicación especiali$z a d a$, Berna, Peter Lang, pp. 273-295.

Myers-Scotton, Carol (1993), Social motivations for codeswitching. Evidence from Africa, Oxford, Clarendon Press.

Pratt, Chris (1980), El anglicismo en el español peninsular contemporáneo, Madrid, Gredos.

Romaine, Suzanne (1995), Bilingualism, 2a . ed., Oxford, Blackwell. 
Sanou, Rosa María, Graciela Albiñana, Claudia CastaÑEDA y GRACIELA Galli (2012), "Nivel socioeducativo y uso de anglicismos en San Juan", en Yolanda Hipperdinger (ed.), Cuestiones lexicológicas y lexicográficas, Mendoza, Sociedad Argentina de Lingüística (en prensa). SARtori, Giovanni (1982), Teoria dei partiti e caso italiano, Milán, Sugar.

Seco, Manuel (1977), "El léxico de hoy”, en Rafael Lapesa (coord.), Comunicación y lenguaje, Madrid, Karpos, pp. 183-201.

Thomason, Sarah Grey y Terrence Kaufman (1988), Language contact, creolization, and genetic linguistics, Los Ángeles, University of California Press.

Torres Cacoullos, Rena y Neddy A. Vigil (2006), "Sustantivos de origen inglés en discurso español: ¿cuál es su gramática?", Rio Bravo. A Journal of the Borderlands, 2, pp. 69-84.

T'Sou, Benjamín (2001), “Language contact and lexical innovation", en Michael Lackner, Iwo Amelung y Joachim Kurtz (eds.), New terms for new ideas, Leiden, Koninklijke Brill, pp. 35-56.

WALlon, Henri (1941), L'évolution psychologique de l'enfant, París, Colin.

Winford, Donald (2003), An introduction to contact linguistics, Londres, Blackwell. 